\title{
EVALUATION OF FARMERS' OSH HAZARD IN OPERATIONNEARBY MOBILE TELEPHONE RADIO BASE STATIONS
}

\author{
Simone Pascuzzi, Francesco Santoro \\ University of Bari Aldo Moro, Italy \\ francesco.santoro@uniba.it
}

\begin{abstract}
In this paper, the authors investigated in the farmers' EMF exposures when they operate in the vicinity of mobile telephone RBSs in order to assess if in the operating frequency range of a transmitting station used by mobile phone service the limits imposed by the Italian laws are kept into proper consideration. Three mobile telephone RBSs having different geometrical and technical features, placed on rural areas of the Apulia Region have been considered. By means of a series of parallelepipeds constructed using a piece of software specifically designed it is possible to state that effective electric field that each of the three investigated stations produced was such that the exposure levels to which farmers are subjected in the surrounding SRB areas are safely within the Italian law limits.
\end{abstract}

Keywords: risk assessment, electromagnetic field, cellular network, farmers' exposure, EC Directive 2013/35.

\section{Introduction}

The agricultural sector is one of the professional activities most affected by injuries also because several operations, such as pruning, pesticide treatments, harvesting, and so on, still require the direct man's involvement, which is therefore subject to specific risk factors [1-3]. In some cases farmers can also operate on agricultural areas contaminated by potential risk situations without their awareness [4-5] as when they are involved in activities both in open-field and within enclosed productive structures in the surrounding areas of radio base stations (RBSs) [6-7]. These RBSs can be placed close to crops that require a lot of activities (i.e. horticultural cultivations, "tendone" trained vineyards, olive orchards, and so on), so that workers, each day in which they perform their job activity, spend a considerable number of hours in their neighbourhood, with no kind of preventive measure to protect against the possible dangerous effect related to the presence of an electromagnetic field [8-9].The irradiation of signal takes place by means of time-varying of both electric field and magnetic field, which permits the transmission of a given amount of energy using electromagnetic waves [10-11]

The Italian Safety Consolidation Act[12]among the various risks for workers' safety included also the risk related to the exposition, during the work, to electromagnetic fields $(0 \mathrm{~Hz} \div 300 \mathrm{GHz})$. This law concerns the safety and health risk protection against the known short term dangerous effects related to induced current circulation and energy absorption inside the human body and arises by the transposition of the ICNIRP recommendations [13] and the EU Directive[14]into national legislation, and their applications were at first established on 30 April 2008 and afterwards delayed by further Directives [15-16]. The exposure limits to EMF for the general public in the frequency range $100 \mathrm{kHz}$ to $300 \mathrm{GHz}$ have been also implemented into Italian legislation [17], according to the ICNIRP guidelines.

In this paper the authors investigated the farmers' exposures who perform their job in the vicinity of mobile telephone RBSs, to assess if the limits imposed by the Italian laws are kept into proper consideration. The farm workers have to be considered like general public. Three mobile telephone RBSs having different geometrical and technical features, placed on rural areas of the Apulia Region (Italy), have been considered. Using the technical data provided by the owners of the RBSs and an adhoc forecast software, the respective radiation patterns were analysed in order to estimate the "lowest distances" beyond which the exposures are below the relevant limits. Some in-situ measurements were also carried out in the neighbourhood of each RBS and the registered values were compared with the threshold safety ones.

\section{Materials and methods}

Theoretical aspects.

Italian legislation [17], providestwo limit values: "Exposure Limits" (that it is not possible to overcome) and "Attention Values" (safe approach against possible effects related to frequently repeated exposure to electromagnetic field, which lasts more thsn 4 hours in a single working day). 
These limits are much lower than the ones pertinent to the occupationally exposed workers, because are referred to individuals unaware of this kind of hazard. Referring to the frequencies normally used by mobile phones, the values of $E=20.0 \mathrm{~V} \cdot \mathrm{m}^{-1}$ and of $E=6.0 \mathrm{~V} \cdot \mathrm{m}^{-1}$ for electrical field intensity and the values of $H=0.05 \mathrm{~A}^{-1} \mathrm{~m}^{-1}$ and of $H=0.016 \mathrm{~A}^{-1}$ for magnetic field are used as "Exposure Limits" (the higher ones) and as "Attention Values" (the lower ones) [10]

Point-to-area transmission is the generic name given to the way in which base stations for mobile communications provide coverage to a given area and the two major types of point-to-area transmission are broadcasting (radio and television) and digital mobile communications (such as GSM and UMTS). The simplest form of RBS antenna used is an "omni-directional" half-wave dipole, which, if mounted with the wire of the antenna vertical, in the horizontal plane, has a radiation pattern(numerical and/or graphical representation of aerial radiated power as a function of azimuthal direction centred on the antenna)uniform in every direction. Generally, however, in order to have a better efficiency, in the RBS construction it is common to use "sectored" or directional panel antennas, that are made by a series of back-shielded radiating elements disposed in a column. This arrangement is intended to produce a pattern, which is asymmetrical, exhibiting (at $-3 \mathrm{~dB}$ gain) not a great wideness on a vertical plane (usually from $5^{\circ}$ to $15^{\circ}$ ) and a greater one on an horizontal plane (usually from $60^{\circ}$ to $90^{\circ}$ ), as reported, for example, in Fig. 1.
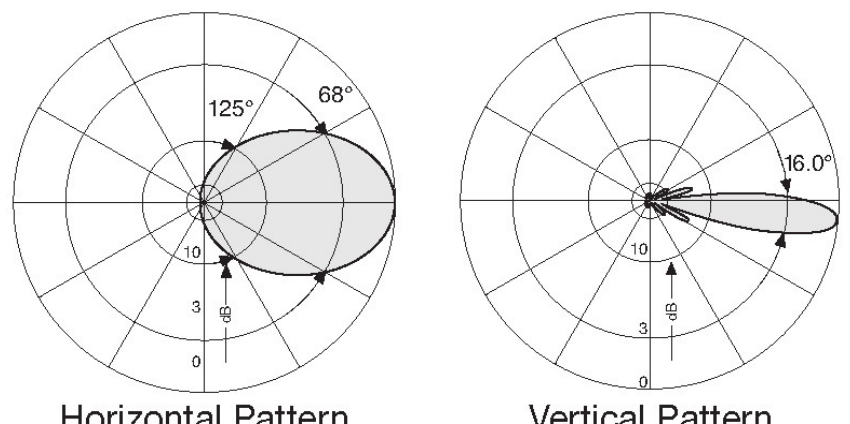

Vertical Pattern

Fig. 1. Typical horizontal and vertical patterns concerning a digital mobile-RBS antenna

The height above the ground of the aerial radio electric centre(RECh, $\mathrm{m}$ ) and its down tilt angle (Down Tilt, degrees) are the parameters that characterize the actual RBS installations (Fig. 2).

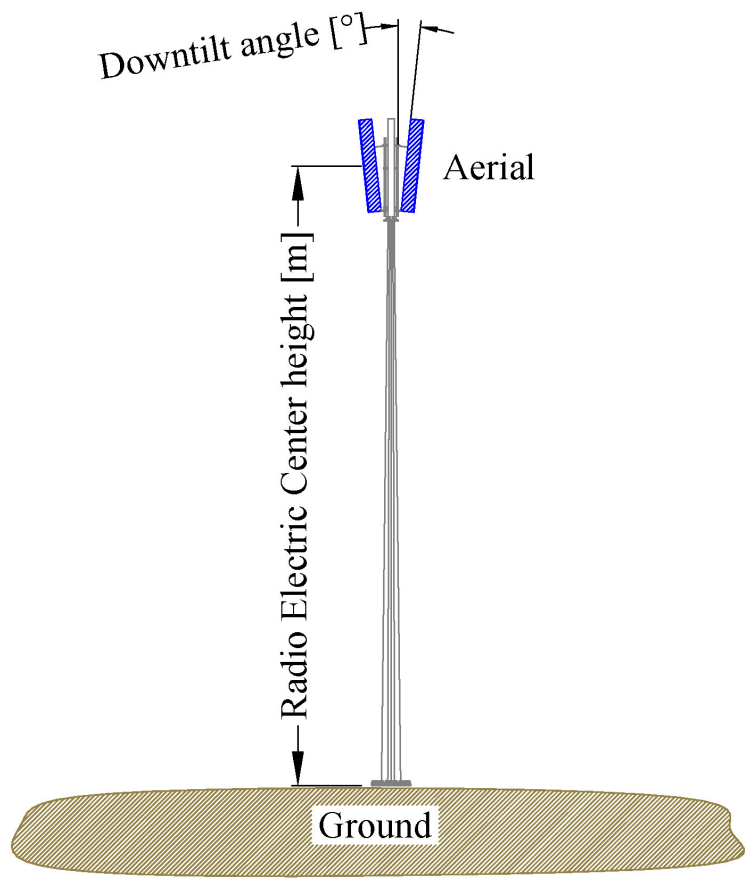

Fig. 2. Typical parameters of an RBS antenna

The EMF produced by an antenna changes its peculiarities with the distancefrom the aerialand, moving away for the aerial itself, it can be differentiated into three main regions: i) reactive near field; 
ii) radiating near field, that is the "Fresnel region"; and iii) the far field, known as the "Fraunhofer region", which extends to infinity. The far field region is the most important one as this determines the antenna's radiation pattern, which does not change shape with distance. This region is characterized by electric radiated field $(\mathrm{E})$, magnetic radiated field $(\mathrm{H})$ and propagation direction orthogonal to each other as well as by plane propagation waves [18]. The power $P_{t}(\mathrm{~W})$ entering the antenna from its connector feed, once it leaves the antenna, creates a power density $P_{d}\left(\mathrm{~W} \mathrm{~m}^{-2}\right)$ in the space. Starting from the concept of "isotropic antenna", which is a theoretical antenna that radiates power equally in every direction (isotropically), it is possible to estimate the power density $P_{d}$ that arises from real antennas, which are passive devices that, due to their directivity, concentrate the power in particular directions (phenomenon known as antenna gain):

$$
P_{d}=\frac{P_{t} \cdot 10^{\frac{G_{t}}{10}}}{4 \pi r^{2}},
$$

where $r-$ is the distance from the aerial, $\mathrm{m}$;

$G_{t}-$ the gain of the antennacompared to an isotropic reference aerial, $\mathrm{dB}_{\mathrm{i}}$.

An alternative, which is commonly used when investigating broadcast antennas, is to consider the electric field strength taking into account that there is a direct link between the power density and the electric field strength. The energy in a radio signal travels as an electromagnetic wave and its main components are, unsurprisingly, an electric field $E\left(\mathrm{~V} \cdot \mathrm{m}^{-1}\right)$ and a magnetic field $H\left(\mathrm{~A} \cdot \mathrm{m}^{-1}\right)$. The power density, in term of magnitude considering the "intrinsic impedance" of free space, is then given by the following equation:

$$
P_{d}=E \cdot H
$$

The ratio of the electric and magnetic fields in an electromagnetic wave is constant in free space and for the far field region (and, as far as we are concerned, in the air); the ratio $E / H$ in free space is called the "intrinsic impedance" of free space. Therefore, in free space:

$$
E=120 \cdot \pi \cdot H
$$

Taking into account the equation (1) and equation (2), the equation (3) can be rearranged in the following manner:

$$
E=\frac{1}{r} \sqrt{30 \cdot P_{t} \cdot 10^{\frac{G_{t}}{10}}}
$$

The EMF values generated by an RBS antenna can be estimated in far field region using this meaningful formula, considering free field conditions, and disregarding phenomena such as the reflections from the soil and, if present, from the infrastructure and the vegetation and far away from the transmitting antenna. This simplified calculation procedure in almost all cases leads to an overestimation of the field values and then is useful in precautionary terms.

\section{The considered RBS antennas}

The considered mobile telephone RBSs have been designed to operate in both $2 \mathrm{G}$ (GSM) and $3 \mathrm{G}$ networks (UMTS). Theyare located inside the rural territory respectively of Giovinazzo (RBS1), Barletta (RBS2), and Peschici (RBS3). These RBSs differ mainly for the transmitting powers with, anyway, a global transmitter powers always less than $100 \mathrm{~W}$ and for the radio electric centre height above the soil, whereas the aerial Down tilt angles were the same $\left(0^{\circ}\right)$ (Table 1).

The aforesaid RBSs had different sector aerial systems, set with different arrangements. Really, RBS1 had two sectors operating in the GSM 900 System; whereas RBS2 was equipped with two sectors operating in the GSM 900 System and another one operating in the GSM 1800 System. The orientation (azimuth) of this last sector matched with a sector pertinent to the GSM 900 system. At last, RBS3 had three sectors operating in the GSM 900 System and two in UMTS system. The azimuths of these two last sectors matched with the azimuth of two sectors pertinent to GSM 900 (Table 2). 
Main technical characteristics of the analyzed RBS

Table 1

\begin{tabular}{|c|c|c|c|c|c|}
\hline Characteristic & RBS1 & \multicolumn{2}{|c|}{ RBS2 } & \multicolumn{2}{c|}{ RBS3 } \\
\hline System & GSM 900 & GSM 900 & GSM 1800 & GSM 900 & UMTS \\
\hline Sector number & 2 & 2 & 1 & 3 & 2 \\
\hline Sector azimuth, degrees & $50-100$ & $110-280$ & 110 & $80-180-300$ & $80-300$ \\
\hline Global transmitter power, W & 91.6 & 56.9 & 43.5 & 54.1 & 19.2 \\
\hline Transmitters number & 8 & 9 & 3 & 9 & 4 \\
\hline RECh, m & 18 & 24 & 24 & 30 & 30 \\
\hline Down tilt, degrees & 0 & 0 & 0 & 0 & 0 \\
\hline \multirow{2}{*}{ Aerial type } & Kathrein & Kathrein & Kathrein & Kathrein & Kathrein \\
\cline { 2 - 6 } & K742271V03 & K730376 & K742235 & K730376 & K742271V01 \\
\hline Aerial gain $(\mathrm{dBi})$ & 16.5 & 18.5 & 19.2 & 18.5 & 17.0 \\
\hline
\end{tabular}

Table 2

Field measurements

\begin{tabular}{|c|c|c|c|c|c|c|}
\hline \multirow{2}{*}{ RBS } & \multirow{2}{*}{$\begin{array}{l}\text { RECh } \\
(\mathrm{m})\end{array}$} & \multicolumn{3}{|c|}{ Sector } & \multirow{2}{*}{$\begin{array}{l}\text { Distances from } \\
\text { RBS, m }\end{array}$} & \multirow{2}{*}{$\begin{array}{c}\text { Measured E-Field, } \\
\mathrm{V} \cdot \mathrm{m}^{-1}\end{array}$} \\
\hline & & GSM 900 & GSM 1800 & UMTS & & \\
\hline \multirow[t]{6}{*}{1} & \multirow{6}{*}{18} & 1 & & & 20 & 1.85 \\
\hline & & & & & 35 & 0.95 \\
\hline & & & & & 70 & 0.41 \\
\hline & & 2 & & & 24 & 1.69 \\
\hline & & & & & 50 & 0.77 \\
\hline & & & & & 70 & 0.30 \\
\hline \multirow[t]{6}{*}{2} & \multirow{2}{*}{24} & 1 & 1 & & 18 & 1.63 \\
\hline & & & & & 42 & 0.48 \\
\hline & & & & & 60 & $*$ \\
\hline & & 2 & & & 15 & 1.34 \\
\hline & & & & & 33 & 0.61 \\
\hline & & & & & 60 & $*$ \\
\hline \multirow[t]{9}{*}{3} & 30 & 1 & & 1 & 20 & 1.38 \\
\hline & & & & & 34 & 0.51 \\
\hline & & & & & 70 & $*$ \\
\hline & & 2 & & & 18 & 1.21 \\
\hline & & & & & 35 & 0.62 \\
\hline & & & & & 70 & 0.34 \\
\hline & & 3 & & 2 & 20 & 1.42 \\
\hline & & & & & 35 & 0.57 \\
\hline & & & & & 75 & $*$ \\
\hline
\end{tabular}

\section{Numerical evaluations}

Using the technical and geometrical characteristics provided by the owner, the analysed RBSs were simulated supposing the use ofthe latest equipment produced by a leading brand in the sector. The EMF produced by these RBSs were forecasted by means of the latest revision available of "NFA3D", which is a specifically crafted piece of software made by "Aldena Telecomunicazioni ltd" designed to fulfil the pertinent Italian Standard [19-20]. The global E-field induced by each RBS is obtained by means of RMS of the E-field induced in each transmitting frequency [21].

The used code allowed to assess the radiation pattern and the solid surfaces, which surround the RBS, characterized by the E-field values that match with the threshold values provided for general public. These lobe-shaped solids, the surfaces of which are the locus of points with the same electric field value equal to $6.0 \mathrm{~V} \cdot \mathrm{m}^{-1}$ ("Attention Values") and $20.0 \mathrm{~V} \cdot \mathrm{m}^{-1}$ ("Exposure Limits"), have been approximated with the corresponding external tangent parallelepipeds for an easier evaluation of the "lowest distances" from the RBS to comply with the Italian legislation. Considering that in this study 
the variability of the electric field was evaluated at the height of $1.70 \mathrm{~m}$ on the ground level (mean height of the human head), the workers' exposures are surely below the relevant limits if beyond these distances.

\section{Field measurements}

According to the technical Standard, for field testing an instrumentation made by PMM (a brand of Narda Safety Test Solutions) has been used consisting of a PMM8053B tester (5 Hz to $40 \mathrm{GHz}$ frequency range; $3 \cdot 10^{-2}-10^{5} \mathrm{~V} \cdot \mathrm{m}^{-1}$ E-field operating range with $10^{-2}-10^{2} \mathrm{~V} \cdot \mathrm{m}^{-1}$ resolution and $0.1-1.0 \mathrm{~V} \cdot \mathrm{m}^{-1}$ sensitivity; $10^{-9}-10^{-2} \mathrm{~T} \mathrm{H}$-field operating range with $10^{-10}-10^{-4} \mathrm{~T}$ resolution and $10^{-8}-10^{-4}$ T sensitivity) and a EP645 isotropic probe $\left(100 \mathrm{kHz}-6.5 \mathrm{GHz}\right.$ frequency range; $0.35-450 \mathrm{~V} \cdot \mathrm{m}^{-1} \mathrm{E}$-field operating range with $0.01 \mathrm{~V} \cdot \mathrm{m}^{-1}$ resolution and $0.35 \mathrm{~V} \cdot \mathrm{m}^{-1}$ sensitivity). The field testingallowed to measure, on a wide range band basis, the values of electric field moving away from the RBSs in each radiating sector direction [22].

According to the Italian Technical standard, during the tests, the instrumentation was located on a nonmagnetic stand by means of which the E-field measurements were carried out at the height of $1.70 \mathrm{~m}$ on the ground level (mean height of the human head) [19], furthermore, the data acquisition lasted for a period of uninterrupted 180 seconds. The root mean square value of the measured data was taken as the final electric field value[20]. In planning the field test, particular care was given in considering the lowest usage rate of mobile phone in non-urban areas if compared to urban ones [13] and during week-end if compared to working days. [23]: the field test took place on working days daytime which can be considered the periods with a much more extensive phone usage [21-22].

\section{Results and discussion}

\section{Theoretical aspects}

Fig. 3 shows the electric field due to the aerial pertinent to RBS1, in its maximum radiation direction (Table 1), considering the gain of $16.5 \mathrm{~dB}_{\mathrm{i}}$ and the peak transmitter power of the sector as of $50 \mathrm{~W}$. The attention value $\left(6 \mathrm{~V} \cdot \mathrm{m}^{-1}\right)$ was calculated $42 \mathrm{~m}$ horizontally far away from the antenna. Taking into account that the Down Tilt of the RBS1 is $0^{\circ}$, this distance is evaluated along a horizontal line passing through the radio electric centre height that is placed at $18 \mathrm{~m}$ above the ground.

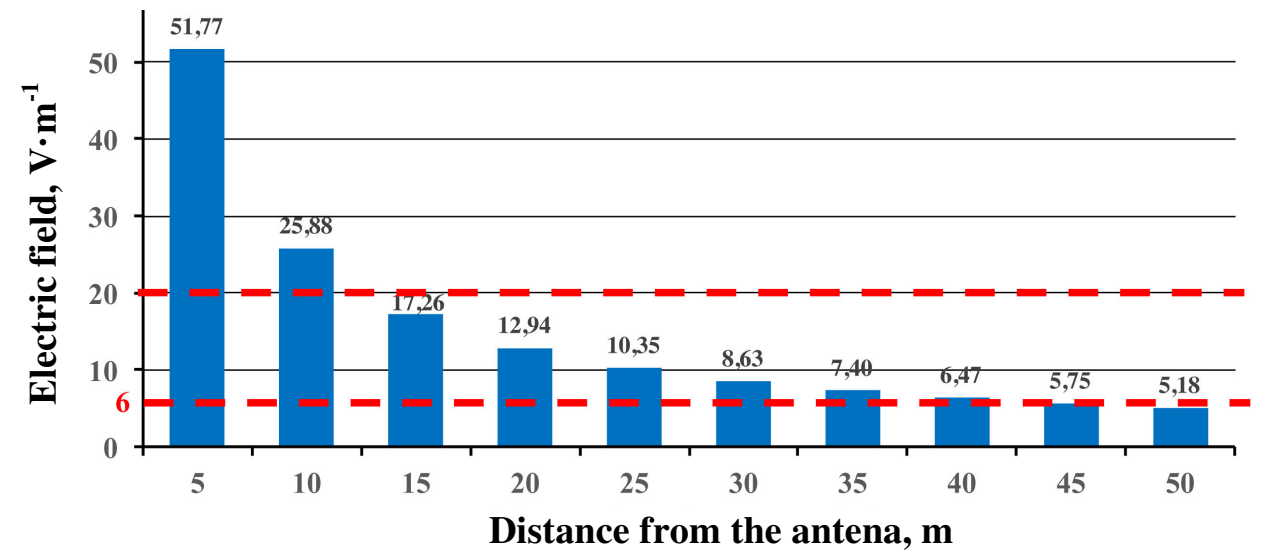

Fig. 3. E-field calculated in the direction of the highest radiation of the RBS antenna (transmitter $50 \mathrm{~W}$, gain $16.5 \mathrm{dBi}$ )

\section{Numerical simulation}

Fig. 4 shows for each examined RBS the parallelepiped externally tangent to the corresponding egg-shaped locus of the points having the same aforesaid E-field limit values.

In particular, the first parallelepiped corresponding to the electric filed value of $20.0 \mathrm{~V} \cdot \mathrm{m}^{-1}$ ("Exposure Limits") is located inside, whereas the second one, offside placed, corresponds to the electric filed value of $E=6.0 \mathrm{~V} \cdot \mathrm{m}^{-1}$ ("Attention Values"). The sizes of each parallelepiped are also reported, related to the reference space coordinate system oriented as reported in the Fig. with the origin placed in the radio electric centre of the respective RBS (Fig. 4). The E-field strength is then higher than $20.0 \mathrm{~V}^{-1}$ inside the first parallelepiped; within the range $6.0-20.0 \mathrm{~V} \cdot \mathrm{m}^{-1}$ in the volume between the first and the second parallelepiped, less than $6.0 \mathrm{~V} \cdot \mathrm{m}^{-1}$ beyond this last one (Fig. 4). 


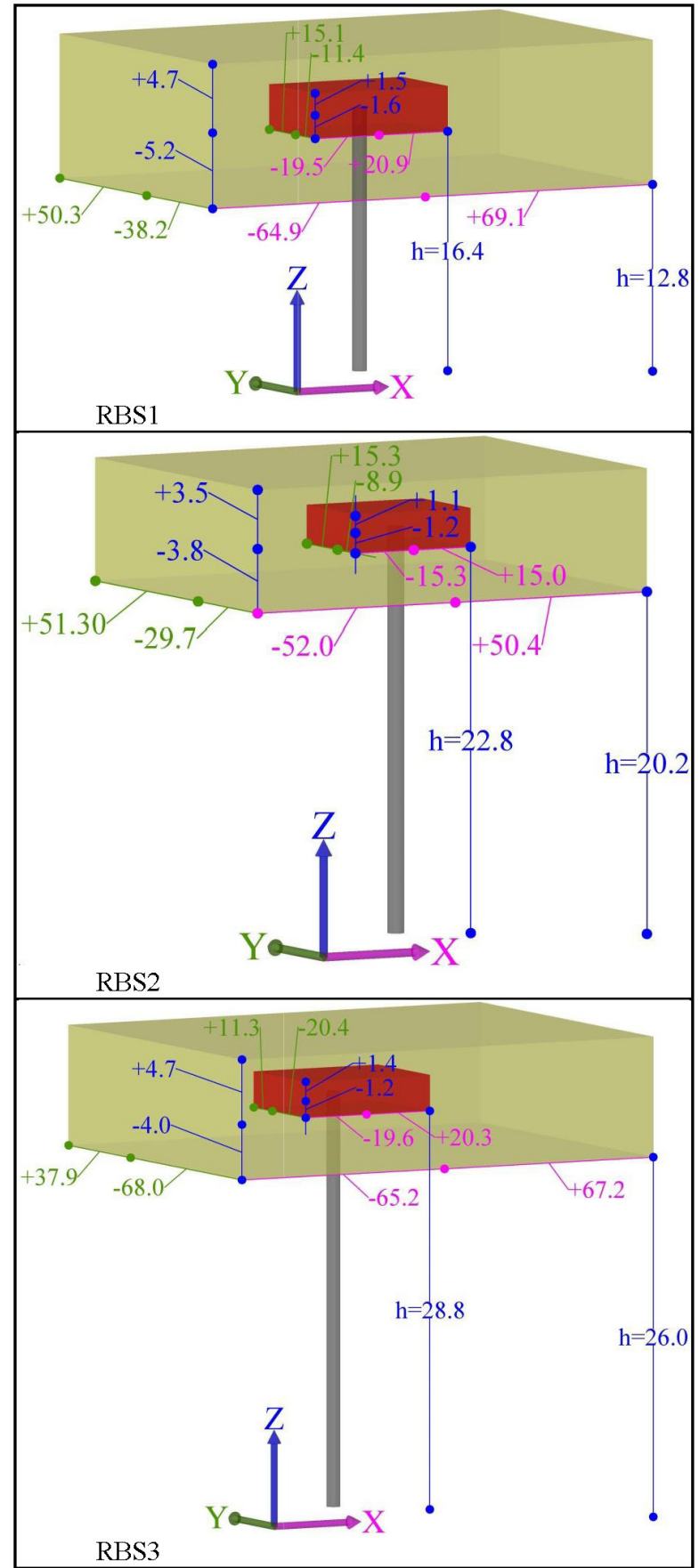

Fig. 4. Parallelepiped externally tangent egg-shaped solids the surfaces of which correspond to E-field limit values

It is evident that all the parallelepipeds corresponding to the "Attention Values" are much over than the mean human head (1.70 $\mathrm{m}$ above the ground), even if these distances are overestimated both because the forecasting was made with the RBSs working at their full capacity and also because the places in which the electric field values are not less than, respectively, $20.0 \mathrm{~V} \cdot \mathrm{m}^{-1}$ and $6.0 \mathrm{~V} \cdot \mathrm{m}^{-1}$, are located according to the azimuth of single RBS antenna sectors, so the major volume inside the aforesaid parallelepipeds is characterized by the electric field values much lower than $20.0 \mathrm{~V} \cdot \mathrm{m}^{-1}$ and $6.0 \mathrm{~V} \cdot \mathrm{m}^{-1}$.

\section{Field measurements}

The field measurements highlight that the effective E-field values are remarkably lesser than the attention values stated by Italian Laws in each aerial sector direction (Table 2). 
Inner parallelepiped $E=20.0 \mathrm{~V} \cdot \mathrm{m}^{-1}$ ("Exposure Limits")-Outer parallelepiped $E=6.0 \mathrm{~V} \cdot \mathrm{m}^{-1}$ (“Attention Values")

In particular, the measured electric field values were below the probe sensitivity (Table 2) for the measurements carried out at the greatest distance from the aerial in the direction of RBS2-Sector 1 and of RBS3-Sector 1\&3. Certainly, also the Down Tilt angle of the aerial sectors has a leading role on the E-field values in the surrounding area. Actually, all the considered RBSs had a DownTilt equal to zero and this operative condition produced a very low E-field at the height of $1.70 \mathrm{~m}$ above the ground. Probably higher E-field values at this height would be measured if the sectors were set with some Down Tilt degrees toward down.

As a further reason for safety, two working characteristics of both $2 \mathrm{G}$ (GSM) and $3 \mathrm{G}$ (UMTS) phone networks have to be considered when a transmission station reaches its maximum output power: the former is designed in order to grant from congestion avoiding further traffic, the latter allows new connection after lowering data rates to the existing connections. In both cases the RBSs are specifically designed not to work at their maximum allowable power for a long time [24]. As a confirmation of this, a study of network measurement data carried out in Sweden, confirmed by in situ measurements, pointed out that only the $10^{\text {th }}$ percentile of average RBSs transmission power evaluated on a whole day period of time for stations located within non-urban areas exceeded the value of the $65 \%$ of the maximum allowable power [24-25].

In this study, the forecasted values of the electric field were, nevertheless, obtained considering the RBSs always working at their maximum allowable transmitting power: this circumstance may only occur if sudden traffic peaks are detected by the RBS management software; in ordinary conditions this does not occur. For all the aforesaid reasons, it is possible to state that during the field tests the transmission power of the station was always considerably below the maximum allowable. In this framework is important to also note that a decrease of about $50 \%$ of the power $P_{t}$ entering the antenna involves taking into account Equation (4) and keeping unchanged the $r$ distance from the aerial a $70 \%$ decrease of the electric field.

\section{Conclusions}

The Radio Base Stations for mobile phone services can, for sure, be considered as low-power sources of electromagnetic fields. The exposure offarm workers when their job takes place nearby a mobile phone Radio Base Station has been analysed considering three different mobile telephone RBSs, placed on rural areas of the Apulia Region (Italy).Taking into account that the farmers are generally unaware of the hazard connected to electromagnetic field exposure and that they are not trained in any kind of preventive measures to protect against the possible dangerous effect related to the presence of an electromagnetic field, this study was carried out in agreement with effective Italian constrain dealing with both occupational and general public exposure to electric and magnetic field varying on a time base. The parallelepipeds built through the numerical simulation and corresponding to the "Attention Values" are much over than the mean human head (1.70 $\mathrm{m}$ above the ground) for all the examined RBSs, even if supposing a full load of the RBS. The effective electric field generated by the considered RBSs was measured by means of field tests and the obtained values were always greatly lower than $6.0 \mathrm{~V} \cdot \mathrm{m}^{-1}$ even nearby the aerials. Even if the experiences reported here refer to a limited number of RBSs and, in order to get better evaluation, a more extensive measurements campaign should be performed, the obtained results point out that for farm workers, who perform their job activity in the surroundings of RBSs, the exposure levels to electromagnetic fields can be considered safely within the Italian Law limits and so the Italian Agency responsible for the protection of the environment (ARPA) could, as it does, safely authorize the construction and the operation of RBSs. Finally, the comparison of the results of the E-field levels with the ICNIRP reference values, clearly shows that the exposure levels are largely below the safety standard limits.

\section{References}

1. Jadhav R., Achutan C., Haynatzki G., Rajaram S., Rautiainen R. Review and Meta-analysis of Emerging Risk Factors for Agricultural Injury. Journal Of Agromedicine, 21(3), 2016, pp. 284-297 
2. DeWit Y., Pickett W., Lawson J., Dosman J. Farm Activities and Agricultural Injuries in Youth and Young Adult Workers. Journal Of Agromedicine, 20(3), 2015, pp. 318-326.

3. Pascuzzi S. A multibody approach applied to the study of driver injures due to a narrow-track wheeled tractor rollover. J. Agr. Eng., 46, 2015, pp. 105-114.

4. Pascuzzi S., Blanco I., Anifantis A.S., Scarascia Mugnozza G. Hazards assessment and technical actions due to the production of pressured hydrogen within a pilot photovoltaic-electrolyzer-fuel cell power system for agricultural equipment. J. Agr. Eng., 47:507, 2016, pp. 89-93.

5. Chiu S., Cheyney M., Ramirez M., Gerr F. Where do agricultural producers get safety and health information? Journal Of Agromedicine, 20 (3), 2015, pp. 265-272.

6. Bianchi B., Tamborrino A., Santoro F. 2013. Assessment of the energy and separation efficiency of the decanter centrifuge with regulation capability of oil water ring in the industrial process line using a continuous method. J. Agr. Eng., 44(S1):E56, 2013, pp. 278-282.

7. Baldoin C., Balsari P., Cerruto E., Pascuzzi S., Raffaelli M. Improvement in pesticide application on greenhouse crops: Results of a survey about greenhouse structures in Italy. Acta Hort. (ISHS) 801, 2008, pp. 609-614.

8. Pascuzzi S. The effects of the forward speed and air volume of an air-assisted sprayer on spray deposition in "tendone" trained vineyards. J. Agr. Eng., 3, 2013, pp.125-132.

9. Pascuzzi S., Cerruto E. An innovative pneumatic electrostatic sprayer useful for tendone vineyards. J. Agr. Eng., 3, 2015, pp.123-127. Pascuzzi S., Santoro F. Exposure of farm workers to electromagnetic radiation from cellular network radio base stations situated on rural agricultural land. International Journal of Occupational Safety and Ergonomics, 21(3), 2015, pp. 351-358.

10. Chatteron P.A. Houlden M.A. 1992. EMC Electromagnetic Theory to practical design. John Wiley and Sons, New York.

11. Law Decree, 2008. Italian Law Decree n.81, April 9, 2008, connected to Law Decree n.106, August 3,2009, concerning the protection of the health and the safety on workplaces.

12. ICNIRP, 2008. "Guidelines for limiting exposure to time-varying electric, magnetic, and EMF (up to $300 \mathrm{GHz}$ )". Published in: HealthPhysics, 1998, 74(4), pp. 494-522.

13. Directive 2004/40/EC. 2004. on the minimum health and safety requirements regarding the exposure of workers to the risks arising from physical agents (EMF).

14. Directive 2013/35/EC.Minimum health and safety requirements regarding the exposure of workers to the risks arising from physical agents (EMF) (20th individual Directive within the meaning of Article 16 (1) of Directive 89/391/EEC) and repealing Directive 2004/40/EC.

15. Law Decree, 2016. Italian Law Decree n.159, August 1, 2016, Implementation of 2013/35/EC.

16. Prime Minister decree, 2003. "Setting of the exposure limits, of the attention values and of the quality goals for the safety of the general public from exposure to produced electric, magnetic and EMF in the frequency range $100 \mathrm{kHz}-300 \mathrm{GHz}$ ". Italian Official Gazette n.199 of August 28,2003

17. Haslett C. 2008. Essentials of Radio Wave Propagation. University press, Cambridge, UK. ISBN 978-0-521-87565-3.

18. CEI 211-7. 2001. "Guide for the measurement and the evaluation of EMF in the frequency range $10 \mathrm{kHz}-300 \mathrm{GHz}$, with reference to the human exposure".

19. CEI 211-10 V1. 2004. "Guide for the realization of a RBS observing the exposure limits to the high frequency EMF"

20. Ardoino L., Barbieri, E., Vecchia, P. Determinants of exposure to electromagnetic fields from mobile phones. Radiation Protection Dosimetry, 111(4), 2004, pp. 403-406.

21. CENELEC, 2008. European Committee for Electrotechnical Standardization. Basic standard for the in-situ measurement of electromagnetic field strength related to human exposure in the vicinity of base stations. CENELEC EN 50492 (2008).

22. Joseph W., Verloock L., Tanghe E. and Martens L. In-situ measurement procedures for temporal RF electromagnetic field exposure of the general public. HealthPhys, 96:5, 2013 pp. 529-542.

23. Mahfouz Z., Gati A., Lautru D., Wong M.F., Wiart J., Hanna V. F. Influence of traffic variations on exposure to wireless signals in realistic. Bioelectromagnetics, 33:, 2011, pp. 288-297.

24. Colombi D., Thors B., Persson T., Wirén N., Larsson L., Jonsson M., Törnevik C. Downlink power distributions for $2 \mathrm{G}$ and $3 \mathrm{G}$ mobile communication networks. Radiation Protection Dosimetry, 157(4), 2013, pp. 477-487. 